\title{
Learning Asynchronous and Sparse Human-Object Interaction in Videos
}

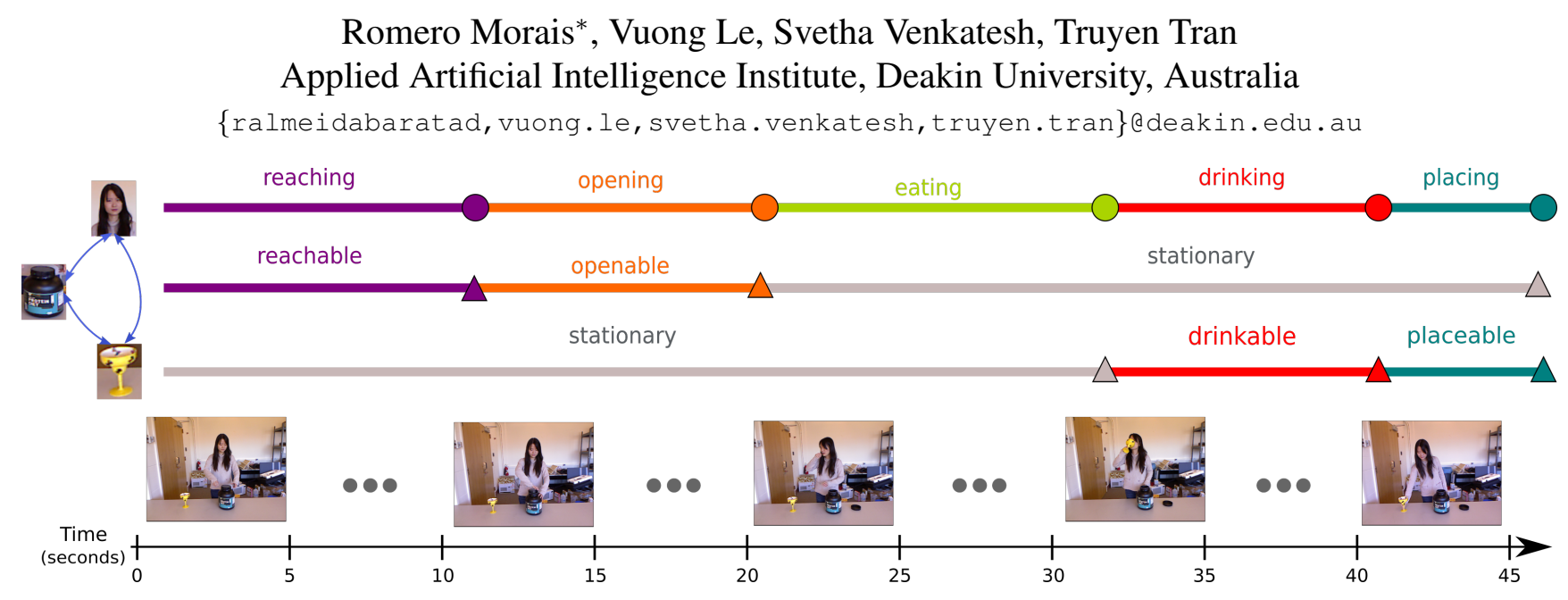

Figure 1: An example of human-object interaction activity, where a person takes some medicine and interacts with two objects. Human (circles) and object (triangles) entities have independent lives throughout the video (upper three rows). Although videos are captured in regular timing (lower rows), the dynamics of human activities and object affordance evolve sparsely and asynchronous with each other (colored segments). They also affect each other (blue curved arrows). These characteristics of human-object interactions are the main modeling goals of this work.

\section{Abstract}

Human activities can be learned from video. With effective modeling it is possible to discover not only the action labels but also the temporal structures of the activities such as the progression of the subactivities. Automatically recognizing such structure from raw video signal is a new capability that promises authentic modeling and successful recognition of human-object interactions. Toward this goal, we introduce Asynchronous-Sparse Interaction Graph Networks (ASSIGN), a recurrent graph network that is able to automatically detect the structure of interaction events associated with entities in a video scene. ASSIGN pioneers learning of autonomous behavior of video entities including their dynamic structure and their interaction with the coexisting neighbors. Entities' lives in our model are asynchronous to those of others therefore more flexible in adaptation to complex scenarios. Their interactions are sparse in time hence more faithful to the true underlying nature and more robust in inference and learning. ASSIGN is tested on human-object interaction recognition and shows superior performance in segmenting and labeling of human sub-activities and object affordances from raw videos. The native ability for discovering temporal structures of the model also eliminates the dependence on external segmentation that was previously mandatory for this task.

\section{Introduction}

Human activities are strongly connected to the surrounding environment and the objects in it. The interactions between human and object entities observed in videos are a fundamental clue toward a deep understanding of human behavior and the surrounding world [8]. This capability is reflected in the human-object interaction (HOI) recognition task, in which human sub-activities (such as "drinking") and object affordances (such as "drinkable") are segmented and recognized from a video by analyzing the interactive relations between them. See Fig. 1 for an example. These relations naturally form a spatio-temporal graph in which entities (humans or objects) and their dynamic interactions evolve throughout the activity. Although entities can be detected and tracked from video, it is challenging to build a graph model that can automatically discover the temporal structure of activities and natively reflect the complex and intricate nature of these interactions.

Currently available approaches applied conditional random field $[14,17,18]$ and graph neural networks $[6,31]$ to model the spatio-temporal entity interaction graph. These models assumed knowledge of the temporal structure of the video, and limited the task to assigning activity and affordance labels to the segments.

Rather than this cascading approach, we exploit the fact that the structure and the content of the events are tightly coupled and may support each other toward the optimal solution in a joint discovery scheme. Such schemes fur- 
ther make possible to break the common assumption that entities in a video are always active and their interactions happen continuously. In reality, unlike regularly captured video frames, interactions between entities happen sparsely in time. This suggests that the temporal relations in the interaction graph can be pruned for a more concise and efficient communication. Furthermore, while bounding boxes are tracked from video frames concurrently, entities' lives are asynchronous with each other. Authentic modeling of this asynchronicity provides a great flexibility to allow entities to act independently and only change their states when necessary.

We introduce Asynchronous-Sparse Interaction Graph Networks (ASSIGN), a joint structure-content discovery framework for sparse and asynchronous human-object interactions. ASSIGN stands on the principle that each entity has an independent life in a video, where it behaves and interacts with its coexisting neighbors in its own pace and timing. This flexible temporal structure and the content labels of events are discovered jointly using two-layer dynamic graph networks that can do inference and be trained end-toend without dependence on external segmentation labels or preprocessing.

The segmentation and labeling capabilities of ASSIGN are demonstrated on two major human-object interaction datasets, with superior quantitative performance and cleaner, more realistic qualitative results than currently available methods. Furthermore ASSIGN shows the unique advantage in modeling the interaction across multiple human entities on the appropriate dataset.

In summary, this paper makes three major contributions:

- Constructs the first end-to-end graph model that jointly learn temporal structure and content label of humanobject interaction activities;

- Effectively models the sparse and asynchronous entities lives in the social context of activity video; and

- Permits efficient relational inference that can skip unnecessary operations resulting it in being robust to a wide variety of event structures.

\section{Related work}

\subsection{Human-object interaction in videos}

Traditional approaches to HOI modeling in videos surround on variations of Markov Random Fields (MRF). Koppula et al. [17] used an MRF to model entities in videos with fully connected spatial and temporal edges. It also starts a trend to use sub-activity segments as temporal time units. This work is extended into the ATCRF model [18] that anticipates future sub-activity/affordances and gathers features from frame-level nodes. ATCRF is further advanced into GP-LCRF [14] to reduce the dimensionality of the frame-level human representation. Another extension of ATCRF is the Recursive CRF [34], in which the CRF is placed under a Bayesian filtering with an efficient belief computation. With the recent advancement of spatiotemporal relation modules, MRF-like models advanced into more efficient implementations with recurrent neural networks (RNNs) and graph neural networks (GNNs). Jain et al. [12] proposed to factorize the dynamic relationships in HOI and model the factors with a mixture of RNNs. Qi et al. [31] proposed Graph Parsing Neural Network (GPNN), which allows spatial graph topology to be inferred adaptively. Ghosh et al. [6] extended GNNs with a stacked hourglass network [28] for label prediction. The MRF and GNN families of models are reliable in predicting HOI labels but they cannot perform temporal segmentation themselves and need to resort to an external segmentation (such as dynamic programming) either before or during inference. ASSIGN on the other hand, learns the segmentation in tandem with the segment labeling directly from frame-level features.

Such joint capability is also the goal of a couple of efforts to constrain HOIs with activity grammars borrowed from natural language processing namely Stochastic grammar [29] and Earley tree parser [30]. These constraints improve the ability to learn relationships between the entities, but at the same time limits their flexibility in the process.

Shared between all previous works is the assumption that entities are synchronous and constantly update their states. This oversimplification is unnatural and being a source of practical issues such as over-segmentation. In this work, we directly challenge this assumption by modeling the independent and sparse behavior of the entities.

\subsection{Action segmentation}

Action segmentation is another line of works that share with us the goal of finding temporal structure of activities in video. Notable works include semi-Markov model [35], multi-class SVM on spatial bag-of-words [9], and segmental RNNs [16]. More recently, convolutional neural networks (CNNs) were more dominant as backbone for action segmentation methods [20, 21, 22]. For instance, Farha and Gall [5] proposed a multi-stage CNN (MS-TCN) with and a truncated MSE loss to handle over-segmentation. Different to this line of works where activities are singularly modeled, we explore the interactions between human and objects and consider the relations between human sub-activities and object affordances.

\subsection{Sparse and asynchronous event modeling}

The sparsity and asynchronicity of events have been a modeling goal of the signal processing community. Neil et al. [27] extended the long short-term memory (LSTM) cell [10] formulation with a "time" gate. This gate intro- 


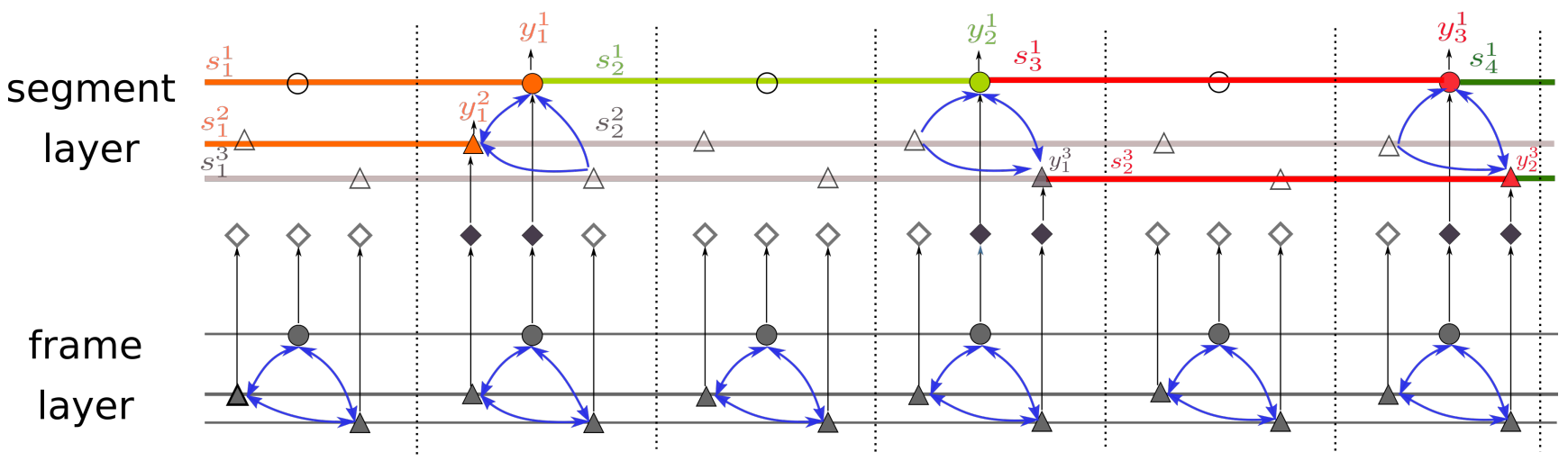

Figure 2: Asynchronous-Sparse Interaction Graph Networks (ASSIGN) architecture contains two layers of spatio-temporal graph networks. At each layer, graph nodes represent human (circle) or object (triangle) entities. Spatial edges are modeled by message passing (blue curved arrows), and temporal edges are modeled through recurrent networks (horizontal lines). The frame level of ASSIGN updates at every time step for every entity, and decides at each step (upward arrows) whether the corresponding segment-level entity change (solid diamond) or skip (hollow diamond) - details in Sec.3.3. The sparse change signals lead to asynchronous and sparse updates (solid shapes) and interactions (blue curved arrows) at the segment level of ASSIGN - details in Sec.3.4. The labels of segments are generated by the second layer at the update operators.

duces "open" and "close" cycles and allows for sparsity in the state updates. Similarly, Campos et al. [2] introduced sparsity into the updates of RNNs by learning binary decisions regularized by a budget loss to skip redundant state updates. In contrast to these works, we skip state updates not only to reduce the computational complexity but also to match the semantics of the human activities. Furthermore, ASSIGN is more advanced in fully using the dense input signal for the sparse activity decisions.

In processing naturally sparse signals, Sekikawa et al. [33] proposed EventNet for real-time asynchronous event streams from event-based cameras. EventNet process events via a two-module architecture timed by the input events and output predictions. Asynchronous data from event-based cameras are also handled by an extended version [24] of the Submanifold Sparse Convolutions (SSC) [7]. This work extend the spatial sparsity modeling of SSC with localized updates throughout the convolutional maps by keeping track of a rulebook per layer. They key difference between this line of work and our formulation is that we explore sparse information from dense signals instead of assuming the signal is already sparse.

\section{Method}

\subsection{Problem formulation}

We are interested in learning the spatio-temporal structures of human-object interactions (HOI) in videos. Previous works consider special cases of a single human [18, 31] or two human hands interacting with multiple objects [4]. We approach this problem in the most generic formulation, where we model an arbitrary number of humans and objects in a video. The problem is defined on a video of $T$ frames with $N$ entities of either human or object class. These entities are detected and their features are extracted from the video. The $e^{t h}$ entity is represented by a temporal sequence of frame-level features $X^{e}=\left\{x_{t}^{e}\right\}_{t=1, \ldots, T}$ together with a class label $c^{e}$. In human-object interaction, this label holds the value of either human or object.

A HOI recognition problem is defined to use input $\left\{X^{e}, c^{e}\right\}_{e=1, \ldots N}$ to generate a temporal segmentation for each entity included. For the $e^{\text {th }}$ entity, the segmentation is of the form $S^{e}=\left\{s_{1}^{e}, s_{2}^{e}, \ldots, s_{n_{e}}^{e}\right\}$ in which each member segment is represented by its start time and end time (which is the start time of next segment) $s_{k}^{e}=\left[t_{k}^{e}, t_{k+1}^{e}\right]$. The output also includes the prediction of segment labels $y^{e}=\left\{y_{1}^{e}, \ldots, y_{n_{e}}^{e}\right\}$ which effectively are sub-activity labels for humans and affordance labels for objects.

For a human entity, a segment label is the name of a subactivity, and for an object entity it is the name of an affordance. These labels are interlinked with each other; for example, a sub-activity "drinking" of a human usually overlaps with affordance "drinkable" of a cup. However, they do not need to be perfectly aligned. An object can remain in the same state during all human activities not involving it. Modeling these sparse and asynchronous relations is the goal of this work.

\subsection{Asynchronous-Sparse Interaction Graph Net- work}

We aim at learning the temporal segmentation and label of sparse events associated with asynchronous entities in a video. To this end, we design a twolayer asynchronous recurrent graph network named Asynchronous-Sparse Interaction Graph Networks 


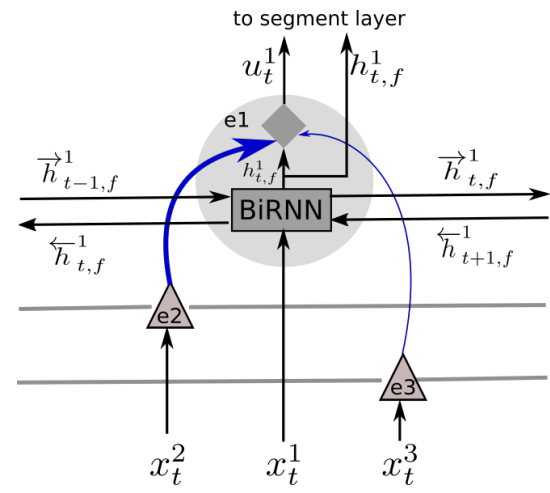

Figure 3: Frame-level node (only enlarged and detailed for human node 1 - the circle) with the BiRNN unit (rectangle) and the segment boundary detector (diamond shape). The detector considers the current state from the recurrent unit and messages from neighboring nodes (blue curved arrows) weighted by an attention mechanism (thickness of arrows). It then makes a decision $u_{t}^{e}$ on whether frame $t$ is the final frame of a segment for each entity. If it is a positive signal $\left(u_{t}^{e}=1\right)$, the summarized context $h_{t, f}^{1}$ is sent up to the segment-level node to predict the label of finalized segment and start a new one.

(ASSIGN). ASSIGN is specialized in modeling each entity in a video (human or object) with two spatio-temporal graphs, one at frame-level and one at segment-level (Figure 2). The frame-level graph nodes process video frames and update their states at every time step, whereas the segment-level nodes update sparsely - only when signaled by the frame-level partner to do so. Each entity decides its own pace asynchronously in the consideration to its neighbors.

\subsection{Segmenting the entity life}

The primary task for ASSIGN is to learn the temporal segmentation of every entity in a video. This translates into making a binary decision at each time step of whether a current segment ends and a new segment starts or not. The segment change of a sub-activity or affordance depends on the internal state of the entity in question and its relation with its neighbors. For example, a human that gets close to a cup makes it a drinkable object. This insight is realized into the design of the frame-level layer of ASSIGN (Fig. 3).

This network takes as input $\left\{X^{e}, c^{e}\right\}_{e=1, \ldots N}$ and builds a spatio-temporal graph. Spatial edges represent interactions between entities, and temporal edges connect instances of the same entity throughout time and represent the internal progression of such an entity. A temporal edge is implemented as a Bidirectional RNN (BiRNN) and generates the hidden state of the $e$-th entity at the $t$-th frame by:

$$
h_{t, f}^{e}=\operatorname{BiRNN}_{f}\left(x_{t}^{e}, \vec{h}_{t-1, f}^{e}, \overleftarrow{h}_{t+1, f}^{e}\right)
$$

where $\vec{h}_{t-1, f}^{e}$ and $\overleftarrow{h}_{t+1, f}^{e}$ are input forward and backward RNN states, and $h_{t, f}^{e}$ is the concatenated output of the two RNNs: $h_{t, f}^{e}=\left[\vec{h}_{t, f}^{e}, \overleftarrow{h}_{t, f}^{e}\right]$

The spatial edges connect different entities at the same time step and reflect the dynamic relations of neighboring entities. It is implemented by pair-wise messaging between entities. We separate the two types of spatial messages: (1) intra-class messages from entities of the same class and (2) inter-class messages from entities of different classes. This separation is important because the nature of the relations are different. For example, the collaboration between two human entities must be modeled differently than the effect of an object on a human.

The frame-level inter-class message to entity $e$ at time $t$ is calculated by:

$$
m_{t, f}^{\text {inter } \rightarrow e}=\operatorname{Att}\left(\left[x_{t}^{e} ; h_{t, f}^{e}\right],\left\{\left[x_{t}^{k} ; h_{t, f}^{k}\right]\right\}_{c^{k} \neq c^{e}}\right) .
$$

Here, Att is the attention operator that calculates the weighted average of the contributions from the neighboring entities. In ASSIGN, it is implemented by a variant of scaled dot-product attention [37] with identical keys and values:

$$
\operatorname{Att}\left(q,\left\{v_{i}\right\}_{i=1, \ldots, n}\right)=\sum_{i=1}^{n} \operatorname{softmax}\left(\frac{q^{T} v_{i}}{\sqrt{d}}\right) v_{i},
$$

where $q$ is a query vector, $\left\{v_{i}\right\}$ is a set of keys/values vectors of size $n$ and, and $d$ is the feature dimension.

Effectively, this operation combines the hidden states $h_{t, f}^{*}$ and inputs $x_{t}^{*}$ of the entities and use them as both keys/values and queries in weighing the relevance of the interacted neighboring nodes (the blue arrows in Fig. 3).

Similarly, the intra-class message is calculated on the set of entities from the same class:

$$
m_{t, f}^{\text {intra } \rightarrow e}=\operatorname{Att}\left(\left[x_{t}^{e} ; h_{t, f}^{e}\right],\left\{\left[x_{t}^{k} ; h_{t, f}^{k}\right]\right\}_{k \neq e, c^{k}=c^{e}}\right) .
$$

These spatial edges resemble a graph attention network [38] except that they dynamically evolve through time.

Finally, we gather the current temporal recurrent state together with the spatial relation messages to make the segmenting decision. This is done by the segment boundary detector (diamond shape in Fig. 3). It contains an MLP $\gamma$ and a differentiable discrete valued estimator using the Gumbel-Softmax (GSM) operator [13, 23]:

$$
u_{t}^{e}=\operatorname{GSM}\left\{\gamma\left(\left[x_{t}^{e} ; h_{t, f}^{e} ; m_{t, f}^{\text {intra }} ; m_{t, f}^{\text {inter }}\right]\right)\right\} .
$$

The binary output $u_{t}^{e}=1$ indicates that $t$ is the last frame of the current segment for the $e$-th entity and $u_{t}^{e}=0$ otherwise. This segmenting signal controls the behavior of the segment-level nodes, which we describe next. 


\subsection{Labeling the learned segments}

The segment-layer of ASSIGN manages the spatiotemporal dynamics of the segments whose boundaries are provided by the frame-layer via the segmenting signal $u_{t}^{e}$ and frame-level state $h_{t, f}^{e}$. This layer is also modeled as a spatio-temporal graph similarly to the frame-layer with BiRNN for temporal edges and attentional message passing for spatial connections.

The key specialty of this graph layer is that its operations are not dense and regular as in the frame layer. Each entity can either update or copy its state, depending on the provided signal $u_{t}^{e}$. This adaptive operation constitutes the asynchronous and sparse behavior of ASSIGN.

At each time step $t$, if $u_{t}^{e}=1$, the node gathers information from the context and update its state using its recurrent operator (blue arrows in the upper half of Fig.2). This includes the segment-level inter-class message

$$
m_{t, s}^{\mathrm{inter} \rightarrow e}=\operatorname{Att}\left(h_{t-1, s}^{e},\left\{h_{t-1, s}^{k}\right\}_{c^{k} \neq c^{e}}\right),
$$

and intra-class message

$$
m_{t, s}^{\text {intra } \rightarrow e}=\operatorname{Att}\left(h_{t-1, s}^{e},\left\{h_{t-1, s}^{k}\right\}_{k \neq e, c^{k}=c^{e}}\right),
$$

where Att is defined in Eq. 3. These messages are calculated similarly to frame-level counter parts in Eqs. 2 and 4. The main distinction is that they are calculated sparsely, only when needed.

We combine these segment-level messages with the frame-level state $h_{t, f}^{e}$ and messages $m_{t, f}^{\text {inter } \rightarrow e}, m_{t, f}^{\text {intra } \rightarrow e}$ previously calculated by the frame layer to form the segmentlevel feature $z_{t}^{e}$ :

$$
z_{t}^{e}=\left[h_{t, f}^{e} ; m_{t, f}^{\mathrm{inter} \rightarrow e} ; m_{t, f}^{\mathrm{intra} \rightarrow e} ; m_{t, s}^{\mathrm{inter} \rightarrow e} ; m_{t, s}^{\mathrm{intra} \rightarrow e}\right] .
$$

This input is fed to the segment-level BiRNN units $\left(\mathrm{BiRNN}_{s}\right)$ to update their states:

$$
h_{t, s}^{e}=\operatorname{BiRNN}_{s}\left(z_{t}^{e}, \vec{h}_{t-1, s}^{e}, \overleftarrow{h}_{t+1, s}^{e}\right)
$$

The updated state is then used to recognize the label of the finished segment:

$$
\hat{y}_{t}^{e}=\operatorname{Softmax}\left(\sigma\left(h_{t, s}^{e}\right)\right),
$$

where $\sigma$ is an MLP and the Softmax is done on appropriate label sets, either human sub-activities or object affordances.

In the other case where $u_{t}^{e}=0$, the node skips a BiRNN update and maintain its current state. This contextualized skipping not only creates sparsity in the state updates but also in the interactions. The inward messages are skipped while outward messages to other updating neighbors can still happen.

This is a better reflection of the world where at-rest entities (e.g. objects far from humans) can avoid unnecessary state updates and over-segmented predictions. It also prevents the short-term memory of the RNNs from fading quickly. Furthermore, operating at the segment-level separate semantic progress (activity) from raw signals (frames) so is more robust to varied video sampling rates. The sophisticated architecture of ASSIGN requires a customized training procedure, which we describe in the next section.

\subsection{Model training}

ASSIGN is effectively a multi-task learning framework where segmentation and labeling tasks are trained together in an end-to-end fashion. It is therefore trained by an ensemble of two losses for the two tasks.

For segmentation, we minimize the binary cross-entropy between a smoothed version of the ground-truth segmentation and the soft output of the boundary detector in Eq. 5

$$
\mathcal{L}_{\text {Seg }}=\frac{1}{T} \sum_{t=1}^{T}\left[\frac{1}{N} \sum_{e=1}^{N} \operatorname{BCE}\left(\hat{\tilde{u}}_{t}^{e}, \tilde{u}_{t}^{e}\right)\right],
$$

where $\hat{\tilde{u}}_{t}^{e}$ is the real value of $u_{t}^{e}$ before binary thresholding and $\tilde{u}_{t}^{e}$ is the smoothed version (with Gaussian filter of $\sigma=$ 4) of the binary pulse ground-truth segmentation.

For labeling, we minimize the negative log-likelihood of the predicted sub-activities and affordance labels:

$$
\mathcal{L}_{\text {Label }}=\frac{1}{T} \sum_{t=1}^{T}\left[\frac{1}{N} \sum_{n=1}^{N} \operatorname{NLL}\left(\hat{y}_{t}^{n}, y_{t}^{n}\right)\right] .
$$

Even though the label is predicted per segment, this loss is calculated per frame so that long segments contribute more than short ones.

The overall loss is the weighted sum of the two losses

$$
\mathcal{L}=\mathcal{L}_{\text {Label }}+\lambda \mathcal{L}_{\text {Seg }},
$$

where $\lambda$ is a tunable parameter.

While the sparsity of ASSIGN operations provide strong advantage in authentic modeling, it creates a subtle obstacle in training where informative gradients from the labeling loss in the segment-layer rarely reaches the frame-layer. This phenomenon makes the frame-layer struggle to have enough training signal to learn adequately. To overcome this issue, we use a two-stage training procedure. In stage 1 , we switch off $\mathcal{L}_{\text {Seg }}$ and set $u_{t}^{e}:=1$ everywhere so that the frame-layer receives a constant stream of directive signals. In stage 2, we turn on the full model and continue to train on top of parameters learned in stage 1 . We observed in our experiments that this two-stage training leads to faster convergence and better final results.

\subsection{Implementation details}

For entity features, we use 2048-dimensional ROI pooling features extracted from the 2D bounding boxes of humans and objects in the video detected by a Faster R-CNN 
[32] module pre-trained [1] on the Visual Genome dataset [19]. We optimize model parameters using the ADAM optimizer [15], with a learning rate of $10^{-3}$. All recurrent networks are built with Gated Recurrent Units (GRU) [3]. The videos are resampled to a uniform 10 FPS frame rate before feeding into the frame-level network. For model validation, we utilize $10 \%$ of the training data as validation data, and select the model with the lowest validation loss during training.

\section{Experiments}

\subsection{Datasets}

We evaluate ASSIGN on the CAD-120 [17] and on the Bimanual Actions [4] datasets. CAD-120 is the most popular dataset for HOI recognition. It contains 120 RGB-D videos of 4 subjects executing 10 different activities, each activity repeated 3 times. Each video is composed of a single person interacting with $1-5$ objects. Sub-activities of the person the affordances of every object are annotated with 10 sub-activity and 12 object affordance labels.

We also experiment on the Bimanual Actions - the first HOI dataset of activities featuring a subject using both hands to interact with objects simultaneously (e.g. left hand holds a nail while right hand hits the nail). It has 540 RGBD videos of 6 subjects conducting 9 different tasks, each task repeated 10 times. The actions of each hand are annotated frame-wise as one of 14 possible actions. For both datasets, we only use the RGB channels to extract frame features.

\subsection{Experimental settings}

We evaluate ASSIGN on two tasks: joint segmentation and label recognition, and label recognition with known segmentation. The first task requires models to segment the time line for each entity in a video and label those segments. The second task is a special case of the first one where the ground-truth segmentation is known and models only need to label the provided segments.

To evaluate how well ASSIGN generalizes to unseen subjects, we do leave-one-subject out cross-validation on both datasets. Previous works concentrated on recognition of labels and usually report frame-level $F_{1}$ scores. However, these metrics are not optimal for tasks involving segmentation because a method might heavily over- or under-segment a video and still attain reasonable framelevel scores. To amend that, we use the $\mathrm{F}_{1} @ k$ metric [20] for the commonly used values of $k=0.10,0.25$, and 0.50 . The $\mathrm{F}_{1} @ k$ metric considers a predicted segment correct if its IoU with the ground-truth segment is at least $k$. Wrong predictions and missed ground-truth segments count as false positives and false negatives, respectively. The $\mathrm{F}_{1} @ k$ is a superior choice over frame-based metrics for joint seg- mentation and labeling problems, and widely used in previous segmentation works $[5,20,26]$. Note that for the label recognition with known segmentation task, $\mathrm{F}_{1} @ k$ is constant for any $k$ and reduced to segment-level micro and macro $F_{1}$ scores. We report these metrics, in line with other reported results in the literature.

\subsection{Quantitative results}

\subsubsection{Joint segmentation and label recognition}

In this main experiment, we compare the performance of ASSIGN with related state-of-the-art and two BiRNNbased baselines on the joint segmentation and label recognition task on the CAD-120 dataset. For this task, the input must be raw video features, with no trace of preproduced segmentation.

Two of the previous works fully qualify for this task: ATCRF [18] and rCRF [34]. Other major related works used the preproduced segmentation information in either explicit or implicit ways. Stochastic grammar [29] used the statistics of segmentation from test portion in training. Earley tree parser [30] repeats the preproduced segment-level features as frame-level features hence implicitly acknowledging the true segment boundaries. More concrete details about these uses are included in the Appendix A.

The baselines are two variations of BiRNN GRU: The Independent BiRNN considers each entity only by its temporal edges, and the Relational BiRNN adds dense spatial connections across entities. (Further details in Appendix C.)

We present the $\mathrm{F}_{1} @ k$ results in Table 1, and in framelevel $F_{1}$ in Appendix D. ASSIGN outperforms both the state-of-the-art and baselines in every configuration of the $\mathrm{F}_{1} @ k$ measure, and for both human sub-activities and object affordances.

This result showcases the advantages of doing segmenting and labeling jointly. Other methods employ separate segmentation and labeling steps, and generate their final result by voting on many different segmentation options. This strategy has a weakness of increasing the over-segmentation when voters disagree and inability to correct if they make the same mistakes.

The BiRNN baselines make frame-wise predictions and lack relational modeling, thus they do not fully leverage the human-object interactions. Despite being simpler, the Independent BiRNN is better than the Relational BiRNN in object affordances. This can be explained by the infrequent changes of object affordances that were mistaken by the presence of dense messages from the human nodes. In contrast, ASSIGN allows sparse messaging and effectively overcomes these problems. 
Table 1: Joint segmentation and label recognition task with no pre-segmentation. Performance on the CAD-120 dataset.

\begin{tabular}{|c|c|c|c|c|c|c|}
\hline \multirow{2}{*}{ Model } & \multicolumn{3}{|c|}{ Sub-activity } & \multicolumn{3}{|c|}{ Object Affordance } \\
\hline & $\mathrm{F}_{1} @ 0.10$ & $\mathrm{~F}_{1} @ 0.25$ & $\mathrm{~F}_{1} @ 0.50$ & $\mathrm{~F}_{1} @ 0.10$ & $\mathrm{~F}_{1} @ 0.25$ & $\mathrm{~F}_{1} @ 0.50$ \\
\hline $\mathrm{rCRF}[34]$ & $65.6 \pm 3.2$ & $61.5 \pm 4.1$ & $47.1 \pm 4.3$ & $72.1 \pm 2.5$ & $69.1 \pm 3.3$ & $57.0 \pm 3.5$ \\
\hline Independent BiRNN & $70.2 \pm 5.5$ & $64.1 \pm 5.3$ & $48.9 \pm 6.8$ & $84.6 \pm 2.1$ & $81.5 \pm 2.7$ & $71.4 \pm 4.9$ \\
\hline ATCRF [18] & $72.0 \pm 2.8$ & $68.9 \pm 3.6$ & $53.5 \pm 4.3$ & $79.9 \pm 3.1$ & $77.0 \pm 4.1$ & $63.3 \pm 4.9$ \\
\hline Relational BiRNN & $79.2 \pm 2.5$ & $75.2 \pm 3.5$ & $62.5 \pm 5.5$ & $82.3 \pm 2.3$ & $78.5 \pm 2.7$ & $68.9 \pm 4.9$ \\
\hline ASSIGN & $\mathbf{8 8 . 0} \pm 1.8$ & $84.8 \pm 3.0$ & $\mathbf{7 3 . 8} \pm 5.8$ & $\mathbf{9 2 . 0} \pm 1.1$ & $\mathbf{9 0 . 2} \pm 1.8$ & $\mathbf{8 2 . 4} \pm 3.5$ \\
\hline
\end{tabular}

Table 2: Label recognition only task with ground-truth segmentation. Performance on the CAD-120 dataset. Unreported results are marked as “-”.

\begin{tabular}{|c|c|c|c|c|}
\hline \multirow{2}{*}{ Model } & \multicolumn{2}{|c|}{ Sub-activity $\mathrm{F}_{1}(\%)$} & \multicolumn{2}{|c|}{ Object Affordance $\mathrm{F}_{1}(\%)$} \\
\hline & Micro & Macro & Micro & Macro \\
\hline GPNN $[31]$ & 76.6 & 72.7 & 74.6 & 54.1 \\
\hline S-RNN (multi-task) [12] & 82.4 & - & 91.1 & - \\
\hline KGS [17] & 86.0 & 80.4 & 91.8 & 81.5 \\
\hline Latent Linear-CRF [11] & 87.0 & 86.0 & - & - \\
\hline ATCRF [18] & 89.3 & 86.4 & 93.9 & 85.7 \\
\hline STGCN [6] & - & 87.2 & - & - \\
\hline ASSIGN & 89.9 & 87.8 & 95.9 & 91.9 \\
\hline
\end{tabular}

Table 3: Joint segmentation and label recognition task with multiple human entities. Performance on Bimanual Actions dataset.

\begin{tabular}{|c|c|c|c|}
\hline \multirow{2}{*}{ Model } & \multicolumn{3}{|c|}{ Sub-activity } \\
\hline & $\mathrm{F}_{1} @ 0.10$ & $\mathrm{~F}_{1} @ 0.25$ & $\mathrm{~F}_{1} @ 0.50$ \\
\hline Dreher et al. [4] & $40.6 \pm 7.2$ & $34.8 \pm 7.1$ & $22.2 \pm 5.7$ \\
\hline Independent BiRNN & $74.8 \pm 7.0$ & $72.0 \pm 7.0$ & $61.8 \pm 7.3$ \\
\hline Relational BiRNN & $77.7 \pm 3.9$ & $75.0 \pm 4.2$ & $64.8 \pm 5.3$ \\
\hline ASSIGN & $84.0 \pm 2.0$ & $81.2 \pm 2.0$ & $\mathbf{6 8 . 5} \pm 3.3$ \\
\hline
\end{tabular}

\subsubsection{Label recognition only}

To examine the sole capability of predicting labels and to match with the task done by more previous works, we setup a simpler experiment where the true segmentation is provided to all methods. This skips the segmentation functionality of ASSIGN and put it in fair comparison with all previous works in their common experimental protocol.

Table 2 presents the micro and macro $F_{1}$ accuracy on CAD-120 dataset. In both metrics and for both sub-activity and object affordance, ASSIGN outperforms all other methods. This further demonstrates that our modeling entities with asynchronous and sparse interactions is a more correct way to label the segments, agnostic to the segmentation quality.

\subsubsection{Multiple human entities}

The generic formulation of ASSIGN makes it easily applied to a wide range of scenarios. We test this capability by trialing it in the case where multiple human entities jointly do a task. This experiment is done on the Bimanual Actions dataset, which contains activities of two hands of the same person interacting with many objects in a shared activity. We compare ASSIGN to the BiRNN baselines (Sec. 4.3.1) and the method of Dreher et al. [4], which is the only previous work proposed for this multi-human setting.

Table 3 compares the performance of these methods on the joint segmentation and labeling task. The method of Dreher et al. [4] has the weakest performance. This can be attributed to their over-simplistic graph network, which ignores the interactions between the two hands and does not take long-term temporal context into account. The BiRNN baselines improve over Dreher et al. [4] by considering longer temporal context but fall short in reaching high accuracy for lacking modeling human-human interactions. ASSIGN makes major improvements over these methods by incorporating cross-hand spatial interaction and asynchronous long-term temporal context. This good performance is also attributed to the segment-level label decisions in contrast to frame-based decisions of the baselines.

Through the quantitative experiments, it is clear that joint structure-content exploration with consideration to the temporal object lives are key features of ASSIGN that makes it excel in recognition performance. The qualitative analysis of outputs and internal operation of the model are examined in the next section.

\subsection{Qualitative analysis}

We compare the detail outputs of ASSIGN and related methods on examples from CAD-120 and Bimanual Actions datasets. Figure 4 shows an example in CAD-120 where ATCRF over-segments human sub-activities. Also, because segments are synchronized between entities, these errors spread to objects and hurt the accuracy on affordance recognition. ASSIGN on the other hand successfully overcomes the over-segmentation and the error propagation by supporting sparse and asynchronous processing.

Figure 5 shows an example of a cooking task on the Bimanual dataset. Dreher et al. [4] take limited temporal context into account and creates many short segments. The Relational BiRNN improves on short segments but fails to handle long ones (e.g. long stir action of the right hand). ASSIGN, with more advanced modeling, is well equipped 


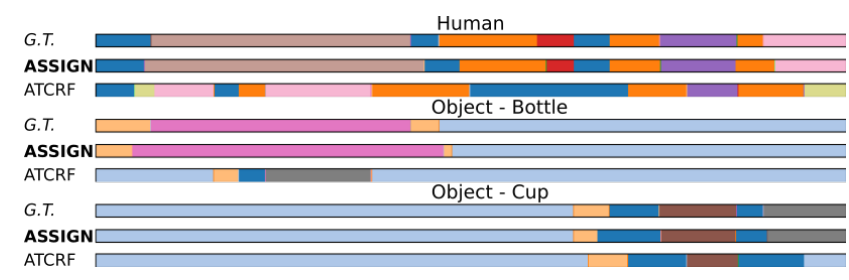

Figure 4: Segmentation and labeling results of the top performing ASSIGN and ATCRF methods compared to ground-truth on the CAD-120 dataset for a taking medicine activity. In this example, ATCRF over-segments the long opening ( $\square$ ) sub-activity for the human. Because objects are synchronized with human in ATCRF, these oversegmentations created a domino effect that leads to the incoherent structure of the timeline of Object - Bottle. In contrast, ASSIGN allow asynchronous state changes of human and objects thus avoided this type of mistakes. Legends: Sub-activities - reaching, opening, moving, eating, drinking, placing, and null; Affordances reachable, openable, stationary, movable, drinkable, and placeable.

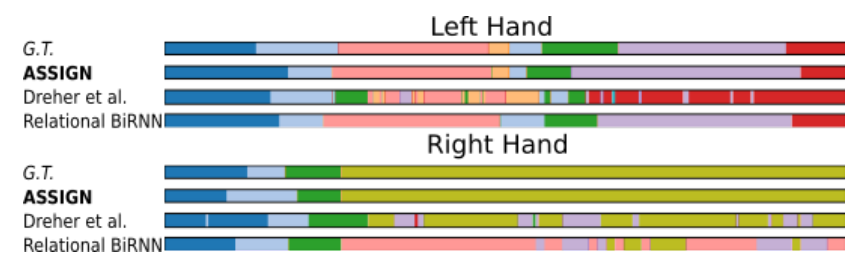

Figure 5: Segmentation and labeling results on the Bimanual dataset for a cooking task. In this example, Dreher et al. [4] creates many spurious short segments due to their model's limited temporal context. The Relational BiRNN baseline improves on short activities, but fails to handle longer events such as the long stir $(\square)$ action because the recurrent memory forgets quickly. On the other hand, ASSIGN handles long actions well by appropriately skipping redundant updates. Legend: $\square$ idle, approach, lift, $\square$ stir, $\square$ hold, $\square$ retreat, $\square$ pour, and $\square$ place.

to reliably handle both short- and long-term interactions.

In Figure 6, we analyze the attention scores of the objects in relation to the human at both levels of ASSIGN. At the frame-level, the human entity is shown to pay sharper attention to a specific object to make crisp decision on transitioning its activity. At the segment level the attention is more uniform. This is reasonable given the sparsity of the updates. At each sparse deciding point, the human entity needs to consider multiple neighboring objects to recognize the label of its sub-activity.

\subsection{Ablation study}

To understand the role of individual components of ASSIGN, we ablate several key modules and evaluate these
Table 4: Ablation study on the CAD-120 dataset.

\begin{tabular}{|c|c|c|c|c|c|}
\hline & \multirow{2}{*}{ Model } & \multicolumn{2}{|c|}{ Sub-activity } & \multicolumn{2}{|c|}{ Object Affordance } \\
\hline & & $\mathrm{F}_{1} @ 0.10$ & $\mathrm{~F}_{1} @ 0.50$ & $\mathrm{~F}_{1} @ 0.10$ & $\mathrm{~F}_{1} @ 0.50$ \\
\hline 1 & w/o message passing & 74.5 & 55.0 & 89.0 & 74.4 \\
\hline 2 & w/o segmentation loss & 84.3 & 69.9 & 89.2 & 78.6 \\
\hline 3 & w/ dense update & 85.5 & 70.3 & 90.6 & 79.8 \\
\hline 4 & w/o pre-training & 87.6 & 71.6 & 91.1 & 78.9 \\
\hline 5 & full ASSIGN model & 88.0 & 73.8 & 92.0 & 82.4 \\
\hline
\end{tabular}

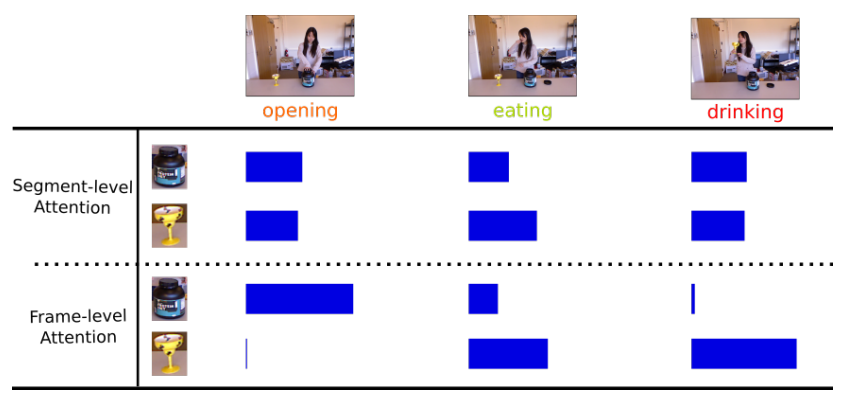

Figure 6: Attention scores of the messages from the objects to the human entity at both layers. Sharp and strong attention on relevant objects are used in frame-level to gather key information. More uniform attention is found in the segment-level where overall consideration is made.

variants on the CAD-120 dataset. Firstly, the spatial message passing has a crucial role in modeling the entity interactions (row 1). On such graph, we join labeling with segmenting tasks by adding the segmentation loss. This loss is also essential for good joint results (row 2). On top of this joint training scheme, ASSIGN is special by using asynchronous and sparse interaction constraints. This innovation is proved to significantly improve the robustness to a wide variety of activity structures (row 3 vs 5). Finally, the strategy of pre-training ASSIGN with the dense model (see Section 3.5) benefits the learning process and supports the model to reach the highest performance (row 4 vs row 5).

\section{Conclusions}

We designed ASSIGN, a two-layer graph network that explores the activity structure concurrently with predicting its content. ASSIGN models human-object interaction more correctly than previous methods by allowing the participating entities to have independent asynchronous lives. The interactions in ASSIGN are sparse, therefore more robust to varied segment lengths and activity progression.

These advantages resulted in superior performance in multiple datasets. Moreover, this performance is consistently strong over larger variations of scenarios than any other method. Deep analysis into ASSIGN's operation shows that the strong performance comes from the new ability to deal with over- or under-segmentation mistakes that previous models suffered from. The generic capabil- 
ity of distilling structure from time series showcases that ASSIGN can be readily applicable to other domains and applications.

\section{References}

[1] Peter Anderson, Xiaodong He, Chris Buehler, Damien Teney, Mark Johnson, Stephen Gould, and Lei Zhang. Bottom-Up and Top-Down attention for image captioning and visual question answering. In 2018 IEEE/CVF Conference on Computer Vision and Pattern Recognition, pages 6077-6086, June 2018. 3.6

[2] Víctor Campos, Brendan Jou, Xavier Giró-i Nieto, Jordi Torres, and Shih-Fu Chang. Skip RNN: Learning to skip state updates in recurrent neural networks. In 6th International Conference on Learning Representations, ICLR 2018, Feb. 2018. 2.3

[3] Kyunghyun Cho, Bart van Merrienboer, Caglar Gulcehre, Dzmitry Bahdanau, Fethi Bougares, Holger Schwenk, and Yoshua Bengio. Learning phrase representations using RNN Encoder-Decoder for statistical machine translation. In Proceedings of the 2014 Conference on Empirical Methods in Natural Language Processing (EMNLP), pages 1724-1734. ACL, 2014. 3.6

[4] Christian R G Dreher, Mirko Wächter, and Tamim Asfour. Learning Object-Action relations from bimanual human demonstration using graph networks. IEEE Robotics and Automation Letters, 5(1):187-194, Jan. 2020. 3.1, 4.1, 3, 4.3.3, 4.4, 5, C, 7, E

[5] Yazan Abu Farha and Jurgen Gall. MS-TCN: MultiStage temporal convolutional network for action segmentation. In 2019 IEEE/CVF Conference on Computer Vision and Pattern Recognition (CVPR), pages 3570-3579. IEEE, June 2019. 2.2, 4.2

[6] Pallabi Ghosh, Yi Yao, Larry Davis, and Ajay Divakaran. Stacked spatio-temporal graph convolutional networks for action segmentation. In The IEEE Winter Conference on Applications of Computer Vision, pages 576-585, 2020. 1, 2.1, 2

[7] Benjamin Graham, Martin Engelcke, and Laurens van der Maaten. 3D semantic segmentation with submanifold sparse convolutional networks. In 2018 IEEE/CVF Conference on Computer Vision and Pattern Recognition, pages 9224-9232, June 2018. 2.3

[8] Abhinav Gupta, Aniruddha Kembhavi, and Larry S Davis. Observing human-object interactions: using spatial and functional compatibility for recognition. IEEE transactions on pattern analysis and machine intelligence, 31(10):1775-1789, Oct. 2009. 1

[9] Minh Hoai, Zhen-Zhong Lan, and Fernando De la Torre. Joint segmentation and classification of human actions in video. In CVPR 2011, pages 3265-3272, June 2011. 2.2

[10] Sepp Hochreiter and Jürgen Schmidhuber. Long shortterm memory. Neural computation, 9(8):1735-1780, Nov. 1997. 2.3

[11] Ninghang Hu, Gwenn Englebienne, Zhongyu Lou, and Ben J A Kröse. Learning latent structure for activity recognition. In 2014 IEEE International Conference on Robotics and Automation (ICRA), pages 1048-1053, May 2014. 2

[12] Ashesh Jain, Amir Roshan Zamir, Silvio Savarese, and Ashutosh Saxena. Structural-RNN: Deep learning on Spatio-Temporal graphs. In 2016 IEEE Conference on Computer Vision and Pattern Recognition (CVPR), pages 5308-5317. IEEE, June 2016. 2.1, 2

[13] Eric Jang, Shixiang Gu, and Ben Poole. Categorical reparameterization with Gumbel-Softmax. In 5th International Conference on Learning Representations, ICLR 2017, Nov. 2016. 3.3

[14] Yun Jiang and Ashutosh Saxena. Modeling HighDimensional humans for activity anticipation using gaussian process latent CRFs. In Robotics: Science and Systems X. Robotics: Science and Systems Foundation, July 2014. 1, 2.1

[15] Diederik P Kingma and Jimmy Ba. Adam: A method for stochastic optimization. In 3rd International Conference on Learning Representations, ICLR 2015, Dec. 2014. 3.6

[16] Lingpeng Kong, Chris Dyer, and Noah A Smith. Segmental recurrent neural networks. In 4th International Conference on Learning Representations, ICLR 2016, Nov. 2015. 2.2

[17] Hema Swetha Koppula, Rudhir Gupta, and Ashutosh Saxena. Learning human activities and object affordances from RGB-D videos. The International journal of robotics research, 32(8):951-970, July 2013. 1, 2.1, 4.1, 2, A, 6

[18] Hema Swetha Koppula and Ashutosh Saxena. Anticipating human activities using object affordances for reactive robotic response. IEEE transactions on pattern analysis and machine intelligence, 38(1):14-29, Jan. 2016. 1, 2.1, 3.1, 4.3.1, 1, 2, 6

[19] Ranjay Krishna, Yuke Zhu, Oliver Groth, Justin Johnson, Kenji Hata, Joshua Kravitz, Stephanie Chen, Yannis Kalantidis, Li-Jia Li, David A Shamma, Michael S Bernstein, and Li Fei-Fei. Visual genome: Connecting language and vision using crowdsourced dense image annotations. International journal of computer vision, 123(1):32-73, May 2017. 3.6

[20] Colin Lea, Michael D Flynn, Rene Vidal, Austin Reiter, and Gregory D Hager. Temporal convolu- 
tional networks for action segmentation and detection. In 2017 IEEE Conference on Computer Vision and Pattern Recognition (CVPR), pages 1003-1012, July 2017. 2.2, 4.2

[21] Colin Lea, Austin Reiter, René Vidal, and Gregory D Hager. Segmental spatiotemporal CNNs for FineGrained action segmentation. In Computer Vision ECCV 2016, pages 36-52. Springer International Publishing, 2016. 2.2

[22] Peng Lei and Sinisa Todorovic. Temporal deformable residual networks for action segmentation in videos. In 2018 IEEE/CVF Conference on Computer Vision and Pattern Recognition, pages 6742-6751, June 2018. 2.2

[23] Chris J Maddison, Andriy Mnih, and Yee Whye Teh. The concrete distribution: A continuous relaxation of discrete random variables. In 5th International Conference on Learning Representations, ICLR 2017, Nov. 2016. 3.3

[24] Nico Messikommer, Daniel Gehrig, Antonio Loquercio, and Davide Scaramuzza. Event-Based asynchronous sparse convolutional networks. In Computer Vision - ECCV 2020, pages 415-431. Springer International Publishing, 2020. 2.3

[25] Romero Morais, Vuong Le, Truyen Tran, Budhaditya Saha, Moussa Mansour, and Svetha Venkatesh. Learning regularity in skeleton trajectories for anomaly detection in videos. In 2019 IEEE/CVF Conference on Computer Vision and Pattern Recognition (CVPR), pages 11988-11996. IEEE, June 2019. B

[26] Romero Morais, Vuong Le, Truyen Tran, and Svetha Venkatesh. Learning to abstract and predict human actions. In Proceedings of the British Machine Vision Conference 2020. British Machine Vision Association, Sept. 2020. 4.2

[27] Daniel Neil, Michael Pfeiffer, and Shih-Chii Liu. Phased lstm: Accelerating recurrent network training for long or event-based sequences. In Advances in Neural Information Processing Systems, pages 38823890, 2016. 2.3

[28] Alejandro Newell, Kaiyu Yang, and Jia Deng. Stacked hourglass networks for human pose estimation. In Computer Vision - ECCV 2016, pages 483-499. Springer International Publishing, Sept. 2016. 2.1

[29] Siyuan Qi, Siyuan Huang, Ping Wei, and Song-Chun Zhu. Predicting human activities using stochastic grammar. In 2017 IEEE International Conference on Computer Vision (ICCV), pages 1173-1181, Oct. 2017. 2.1, 4.3.1, A

[30] Siyuan Qi, Baoxiong Jia, and Song-Chun Zhu. Generalized earley parser: Bridging symbolic grammars and sequence data for future prediction. In Proceedings of the 35th International Conference on Machine Learning, volume 80 of Proceedings of Machine Learning Research, pages 4171-4179, Stockholmsmässan, Stockholm Sweden, 2018. PMLR. 2.1, 4.3.1, A

[31] Siyuan Qi, Wenguan Wang, Baoxiong Jia, Jianbing Shen, and Song-Chun Zhu. Learning Human-Object interactions by graph parsing neural networks. In Computer Vision - ECCV 2018, pages 407-423. Springer International Publishing, 2018. 1, 2.1, 3.1, 2

[32] Shaoqing Ren, Kaiming He, Ross Girshick, and Jian Sun. Faster R-CNN: Towards Real-Time object detection with region proposal networks. IEEE transactions on pattern analysis and machine intelligence, 39(6):1137-1149, June 2017. 3.6

[33] Yusuke Sekikawa, Kosuke Hara, and Hideo Saito. EventNet: Asynchronous recursive event processing. In 2019 IEEE/CVF Conference on Computer Vision and Pattern Recognition (CVPR), pages 3882-3891, June 2019. 2.3

[34] Ozan Sener and Ashutosh Saxena. rCRF: Recursive belief estimation over CRFs in RGB-D activity videos. In Robotics: Science and Systems XI. Robotics: Science and Systems Foundation, July 2015. 2.1, 4.3.1, 1,6

[35] Qinfeng Shi, Li Cheng, Li Wang, and Alex Smola. Human action segmentation and recognition using discriminative Semi-Markov models. International journal of computer vision, 93(1):22-32, May 2011. 2.2

[36] Nitish Srivastava, Elman Mansimov, and Ruslan Salakhudinov. Unsupervised learning of video representations using LSTMs. In Francis Bach and David Blei, editors, Proceedings of the 32nd International Conference on Machine Learning, volume 37 of Proceedings of Machine Learning Research, pages 843852. PMLR, July 2015. B

[37] Ashish Vaswani, Noam Shazeer, Niki Parmar, Jakob Uszkoreit, Llion Jones, Aidan N Gomez, Ł Ukasz Kaiser, and Illia Polosukhin. Attention is all you need. In Advances in Neural Information Processing Systems 30, pages 5998-6008. 2017. 3.3

[38] Petar Veličković, Guillem Cucurull, Arantxa Casanova, Adriana Romero, Pietro Liò, and Yoshua Bengio. Graph attention networks. In 6th International Conference on Learning Representations, ICLR 2018, Feb. 2018. 3.3 


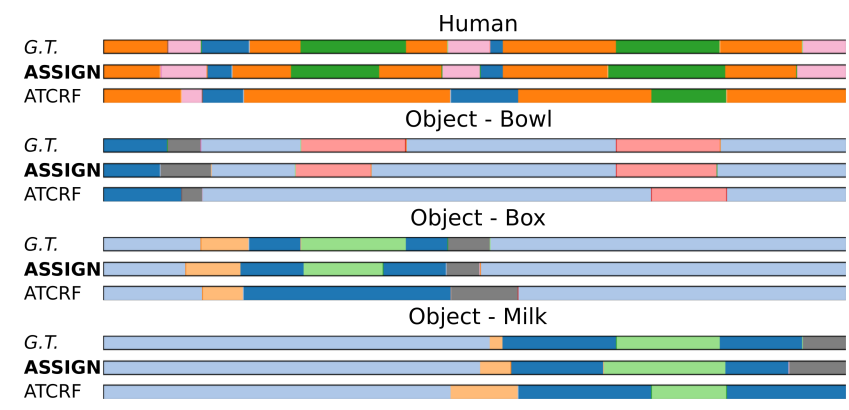

Figure 7: Segmentation and labeling results of ASSIGN and ATCRF on the CAD-120 dataset for a making cereal activity. In this example, ATCRF under-segments the human and the box by skipping a few segments and merging adjacent labels. Sub-activities: moving, placing, reaching, and pouring. Affordances: movable, placeable, stationary, pour-to, reachable, and pourable.

\section{A. Qualification for joint segmentation and la- bel recognition task}

As mentioned in Section 4.3.1, [29, 30] implicitly or explicitly used information relevant to the ground-truth segmentation during the training or testing of their models.

For the Stochastic Grammar method [29], the model requires the computation of data statistics such as length of sub-activities and object affordances, and these were computed from the whole dataset. The experimental protocol is a leave-one-subject out cross-validation, which requires such data-dependent statistics to be computed from the training folds during each round of cross-validation. The code relies on these pre-computed statistics to execute, and in the files provided by the authors in a Github issue ${ }^{1}$, we can verify that the lengths of sub-activities and object affordances provided are in relation to the full dataset.

For the Generalized Earley Parser method [30], the authors implicitly give segmentation information about the data to their model by repeating the segment-level features provided by Koppula et al. [17] as frame-level features. We can confirm that by analyzing the function collate_fn_cad ${ }^{2}$, where the length information about the segments is used in lines 40-44 to assemble the frame-level features. This means that the segmentation is implicitly input to their model, which makes their method not suitable for this task.

\section{B. Training loss implementation details}

In addition to the segmentation and recognition losses, ASSIGN has an anticipation loss. This anticipation loss is identical to the recognition loss, but predicts the label of the next segment. All ASSIGN variations were trained with this

\footnotetext{
${ }^{1}$ https://github.com/SiyuanQi/grammar-activity-prediction/issues/2

${ }^{2} \mathrm{https}: / /$ github.com/SiyuanQi/generalized-earleyparser/blob/master/src/python/datasets/utils.py
}

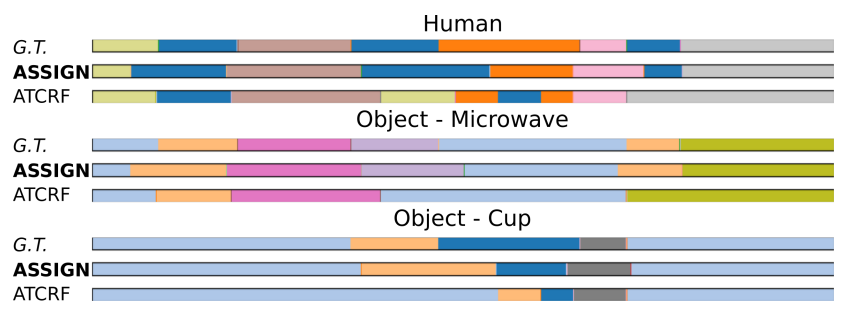

Figure 8: Segmentation and labeling results of ASSIGN and ATCRF on the CAD-120 dataset for a taking food activity. In this example, ATCRF over-segments the moving ( ) sub-activity and ignores the last reaching ( ). ASSIGN correctly segments and label the entities. Sub-activities: null, reaching, opening, moving, placing, and closing. Affordances: stationary, reachable, openable, containable, closeable, movable, and placeable.

Table 5: $\mathrm{F}_{1} @ k$ results for ASSIGN with and without anticipation loss on the CAD-120 dataset.

\begin{tabular}{|c|c|c|c|c|}
\hline \multirow{2}{*}{ Model } & \multicolumn{2}{|c|}{ Sub-activity } & \multicolumn{2}{|c|}{ Object Affordance } \\
\hline & $\mathrm{F}_{1} @ 0.10$ & $\mathrm{~F}_{1} @ 0.50$ & $\mathrm{~F}_{1} @ 0.10$ & $\mathrm{~F}_{1} @ 0.50$ \\
\hline ASSIGN w/o anticipation loss & 86.2 & 71.4 & 91.3 & 80.4 \\
\hline ASSIGN & 88.0 & 73.8 & 92.0 & 82.4 \\
\hline
\end{tabular}

anticipation loss. We show in Table 5 the $\mathrm{F}_{1} @ k$ scores on the CAD-120 dataset for ASSIGN with and without the anticipation loss. Similarly to previous works [25, 36], doing anticipation helps with the recognition results.

\section{BiRNN baselines}

We designed two baseline models for our experiments: the Independent BiRNN and the Relational BiRNN.

The Independent BiRNN is simply a BiRNN per entity (with shared parameters for entities of the same class) followed by an MLP to recognize the sub-activity (or affordance) of the entity. We call it independent because there is no message passing between the entities. More specifically, for the $e$-th entity at the $t$-th frame we compute its BiRNN state as

$$
h_{t}^{e}=\operatorname{BiRNN}\left(x_{t}^{e}, \vec{h}_{t-1}^{e}, \overleftarrow{h}_{t+1}^{e}\right)
$$

where $x_{t}^{e}$ is the frame-level feature for the $e$-th entity at the $t$-th frame, and $h_{t}^{e}=\left[\vec{h}_{t}^{e} ; \overleftarrow{h}_{t}^{e}\right]$ is the concatenation of the forward and backward hidden states produced by the BiRNN. We recognize the label associated with the $t$ th frame as

$$
\hat{y}_{t}^{e}=\operatorname{Softmax}\left(\alpha\left(h_{t}^{e}\right)\right),
$$

where $\alpha$ is an MLP and label recognition is done framewise. 


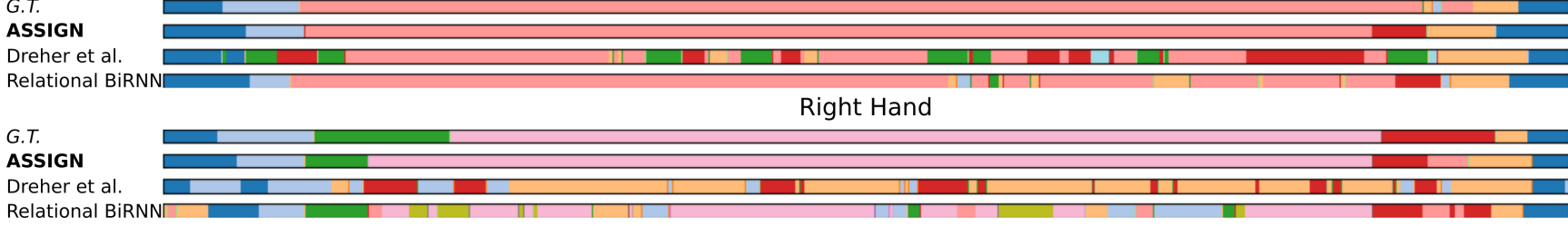

Figure 9: Segmentation and labeling results on the Bimanual Actions dataset for a sawing task. The main difficulty related methods have is to handle long actions, such as the left hand hold ( ). Legend: $\square$ idle, approach, hold, retreat, place, lift, and saw.

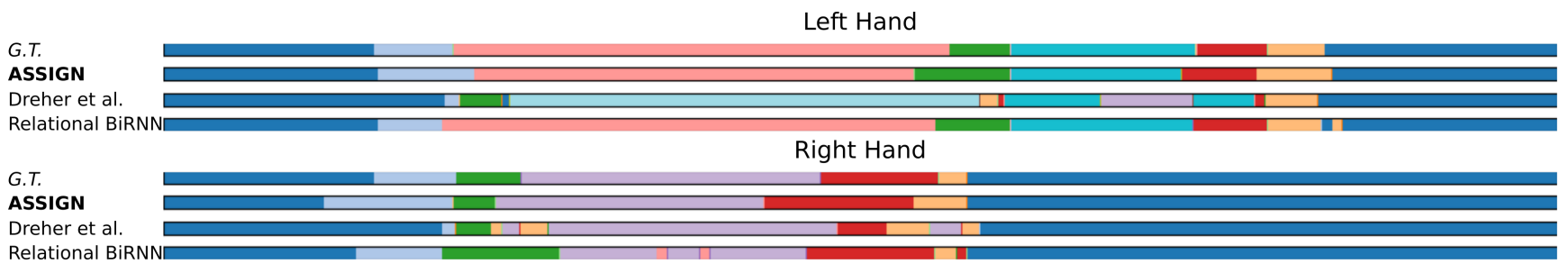

Figure 10: Segmentation and labeling results on the Bimanual Actions dataset for a pouring task. In this example, the Relational BiRNN has results comparable to ASSIGN but still makes several over-segmentation mistakes in both hands, such as the right hand pour $(\square)$. Legend: $\square$ idle, approach, $\square$ hold, $\square$ lift, $\square$ drinking, $\square$ place, $\square$ retreat, and pour.

The Relational BiRNN is similar to the Independent BiRNN, but it includes inter-class messages between the entities. The messages exchanged in the Relational BiRNN are a mean-pooling of the hidden states of the entities of the sender class. We compute the model BiRNN state as

$$
h_{t}^{e}=\operatorname{BiRNN}\left(x_{t}^{e}, \vec{h}_{t-1}^{e}, \overleftarrow{h}_{t+1}^{e}\right)
$$

The message to entity $e$ is an inter-class message only, and we compute it as the average of the hidden states of the entities of class $c^{k}$

$$
m_{t}^{i n t e r \rightarrow e}=\frac{1}{K} \sum_{c^{k} \neq c^{e}} h_{t}^{k},
$$

where $K$ is the number of entities for which $c^{k} \neq c^{e}$. Finally, we recognize the label associated with the $t$-th frame as

$$
\hat{y}_{t}^{e}=\operatorname{Softmax}\left(\alpha\left(\left[h_{t}^{e} ; m_{t}^{\text {inter } \rightarrow e}\right]\right)\right),
$$

where $\alpha$ is an MLP. Since the Bimanual Actions dataset [4] is not annotated with object affordances, we do not include the human $\rightarrow$ object message in its Relational BiRNN.

\section{Frame-level micro and macro $F_{1}$ results}

We report in Section 4.3.1 the $\mathrm{F}_{1} @ k$ metric for the joint segmentation and label recognition task. To provide a complete analysis of ASSIGN and related methods, we include here the micro and macro $\mathrm{F}_{1}$ results on both CAD-120 (Table 6) and Bimanual Actions (Table 7) datasets.
Table 6: Joint segmentation and label recognition task with

\begin{tabular}{|c|c|c|c|c|}
\hline \multirow{2}{*}{ Model } & \multicolumn{2}{|c|}{ Sub-activity $\mathrm{F}_{1}(\%)$} & \multicolumn{2}{|c|}{ Object Affordance $\mathrm{F}_{1}(\%)$} \\
\hline & Micro & Macro & Micro & Macro \\
\hline Independent BiRNN & 58.0 & 54.2 & 83.3 & 73.3 \\
\hline rCRF [34] & 68.1 & 61.3 & 81.5 & 77.8 \\
\hline KGS [17] & 68.2 & 66.4 & 83.9 & 69.6 \\
\hline Relational BiRNN & 70.3 & 67.7 & 81.6 & 66.4 \\
\hline ATCRF [18] & 70.3 & 70.2 & 85.4 & 71.9 \\
\hline ASSIGN & 74.8 & 73.3 & 86.9 & 79.6 \\
\hline
\end{tabular}
no pre-segmentation. Micro and macro $\mathrm{F}_{1}$ performance on the CAD-120 dataset.

Table 7: Joint segmentation and label recognition task with no pre-segmentation. Micro and macro $\mathrm{F}_{1}$ performance on the Bimanual Actions dataset.

\begin{tabular}{cccc}
\hline \multirow{2}{*}{ Model } & & \multicolumn{2}{c}{ Sub-activity $\mathrm{F}_{1}(\%)$} \\
& & Micro & Macro \\
\cline { 1 - 1 } \cline { 4 - 4 } Dreher et al. [4] & & 64.0 & 63.0 \\
Independent BiRNN & & 76.7 & 74.8 \\
Relational BiRNN & & 80.3 & 77.5 \\
\cline { 1 - 1 } \cline { 4 - 5 } ASSIGN & & $\mathbf{8 2 . 3}$ & $\mathbf{7 9 . 5}$ \\
\hline
\end{tabular}

For both datasets, ASSIGN attains superior performance when compared to related methods and baselines. It is interesting to note that the Relational BiRNN has lower macro $\mathrm{F}_{1}$ scores than ATCRF, even though it has higher $\mathrm{F}_{1} @ k$ scores. This relates to the discussion in Section 4.2 that frame-level micro/macro scores are not the most appropriate metric when dealing with joint segmentation and recognition problems. For example, in a situation where a method over-segments a long segment, this might not reflect badly 
on the frame-level metrics but it will reflect badly on the $\mathrm{F}_{1} @ k$ metric since the model effectively splits the long segment into many short segments.

\section{E. Segmentation and labeling extra qualitative comparisons}

We further illustrate the segmentation and labeling results of ASSIGN by showing some more qualitative comparisons between ASSIGN and related methods.

For the CAD-120 dataset, we show a making cereal and a taking food activities in Figures 7 and 8, respectively. For making cereal, we observe that ATCRF under-segments the entities, which can happen to their model whenever their ensembling strategy agrees on a wrong label. For the taking food activity we observe a mixed behavior: ATCRF over-segments halfway through the video, the moving $(\square)$ sub-activity, and under-segments later the closing ( ) subactivity. For both scenarios and entities in them, ASSIGN correctly segments and label the segments.

For the Bimanual Actions dataset, we show a sawing and a pouring activities in Figures 9 and 10, respectively. For both activities, we observe that the biggest hurdle for Dreher et al. [4] and the Relational BiRNN is sustaining the prediction for long actions, which leads them to oversegmentation issues. For example, the long hold ( $\square$ ) and the long saw ( ), in Figure 9, are heavily over-segmented by them. ASSIGN, on the other hand, has no issues with that. 